\title{
Luttinger liquid coupled to Ohmic-class environments
}

\author{
Andisheh Khedri $\odot,{ }^{1}$ Antonio Štrkalj $\odot,{ }^{1}$ Alessio Chiocchetta, ${ }^{2}$ and Oded Zilberberg ${ }^{1}$ \\ ${ }^{1}$ Institute for Theoretical Physics, ETH Zurich, CH-8093 Zurich, Switzerland \\ ${ }^{2}$ Institute for Theoretical Physics, University of Cologne, D-50937 Cologne, Germany
}

(Received 14 December 2020; revised 23 April 2021; accepted 17 May 2021; published 9 July 2021)

\begin{abstract}
We investigate the impact of an Ohmic-class environment on the conduction and correlation properties of one-dimensional interacting systems. Interestingly, we reveal that interparticle interactions can be engineered by the environment's noise statistics. Introducing a backscattering impurity to the system, we address KaneFisher's metal-to-insulator quantum phase transition in this noisy and realistic setting. Within a perturbative renormalization group approach, we show that the Ohmic environments keep the phase transition intact, while sub- and super-Ohmic environments modify it into a smooth crossover at a scale that depends on the interaction strength within the wire. The system still undergoes a metal-to-insulator-like transition when moving from subOhmic to super-Ohmic environment noise. We cover a broad range of realistic experimental conditions, by exploring the impact of a finite wire length and temperature on transport through the system.
\end{abstract}

DOI: 10.1103/PhysRevResearch.3.L032013

Introduction. One of the most fascinating manifestations of quantum many-body physics occurs in one-dimensional systems. There, irrespective of whether the interacting particles are gapless fermions, bosons, or spins, their low-energy properties universally exhibit Tomonaga-Luttinger liquid (TLL) behavior with well-defined bosonic excitations [1-5]. Distinct signatures of TLL [6] include separation of spin and charge degrees of freedom [7-10], which are experimentally verified as the fractionalization of injected charges [11-14]; power-law behavior of correlation functions, also known as the zero-bias anomaly [15-17]; and Kane-Fisher impurity physics [18-20]. The latter concerns the sensitivity of gapless excitations to local perturbations, that are microscopically rationalized in terms of Friedel oscillations [21], and as a manifestation of orthogonality catastrophe [22-24]. Thus, the presence of a backscattering impurity inside a TLL [18,19,25] engenders a quantum phase transition between a perfectly conducting phase and an insulating phase as a function of interaction strength. Such TLL features have been observed in a wide variety of experiments including nanotubes $[16,17,26]$, quantum Hall edges [27,28], cold-atom platforms [29-31], circuit quantum simulations [25], antiferromagnetic spin chains [32], and spin ladder systems [33,34].

Recent technological advances in solid-state platforms $[12,35,36]$, as well as many-body quantum simulators with cold-atom experiments [30,37-41], or quantum circuits $[25,42,43]$, sparked a renewed interest in the dynamics of open quantum systems [44]. Here, the competition between coherent quantum processes and incoherent forcing induced

Published by the American Physical Society under the terms of the Creative Commons Attribution 4.0 International license. Further distribution of this work must maintain attribution to the author(s) and the published article's title, journal citation, and DOI. by the environment leads to novel physics, with no counterpart in isolated quantum systems [45-54], and raises fundamental questions regarding the existence of universality in open quantum systems. As a result, the interplay between TLL physics, dissipation, and drive is also revisited, leading to novel effects, such as a many-body quantum Zeno effect due to localized loss [55-60], engineering correlation with nonlocal two-body loss effects [61,62], exotic phases such as Zeno insulators, and dissipation-induced spin-charge separation [63]. Similarly, the impact of out-of-equilibrium scenarios has been extensively explored [50,64-68], highlighting further universal open TLL physics.

A particularly relevant instance of an open TLL involves the presence of leads (reservoirs) attached to the ends of a quantum wire. This setup is fundamental for exploring quantum transport $[30,41,69,70]$. However, the presence of leads (and their resulting dissipative channels) unavoidably affects the wire's transport properties, where even the presence of noninteracting one-dimensional (Ohmic) leads melts the insulating phase of dirty TLLs [67,71-75], as well as the aforementioned zero-bias anomaly [76]. The leads have shown to have a more dramatic impact on transport and the $I-V$ characteristic of the interacting wire, when they are modeled as TLLs with interaction strengths different from that of the wire [77,78]. Realistic leads, however, are realized by distinctly different systems, ranging from metallic gates with an unscreened Coulomb potential [79] or lattice vibrations [80], to superfluid ultracold gases [81], or to complex $R C$ circuits [82]. Accordingly, these realizations bring about different open system scenarios, with various environment densities of states, coupling to the TLL, and fluctuations. This diversity can result in vastly different phenomena. Despite its criticality, to our knowledge, a unifying, low-energy theory that is able to include the effect of arbitrary reservoirs (that are not necessarily TLLs) coupled to a TLL is still missing. 


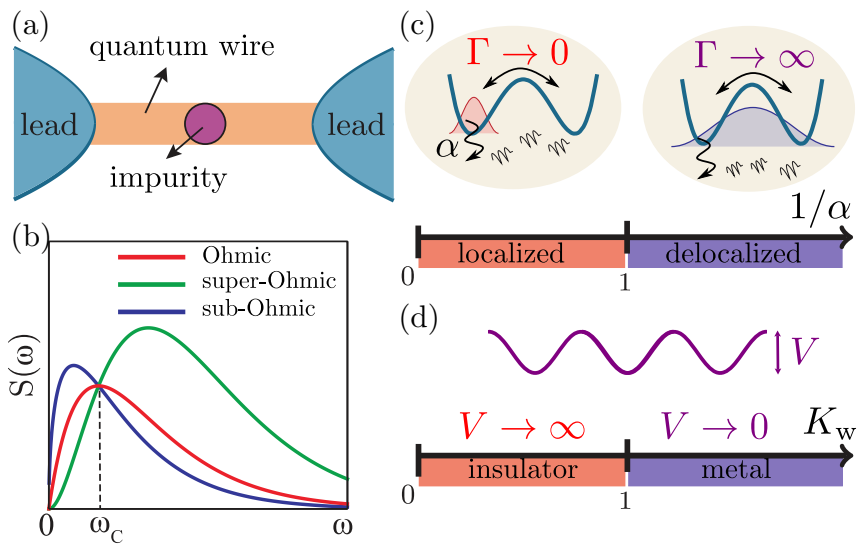

FIG. 1. (a) Sketch of a quantum wire containing a single impurity, that backscatters electrons, coupled to leads. (b) The noisepower spectrum of the electronic leads as a function of frequency, where $\omega_{c}$ marks the environment bandwidth. (c) The phase diagram of a two-level system ( $\phi^{4}$ theory) coupled to an Ohmic environment for varying dissipation strength $\alpha$. For small dissipation $\alpha<1$, the effective tunneling $\Gamma$ between two potential wells diverges, i.e., the particle is delocalized, but for stronger dissipation $\alpha>1$ the particle is localized $\Gamma \rightarrow 0$ [83]. (d) Phase diagram of the TLL hosting an impurity coupled to Ohmic leads [cf. action (4), with an effective scattering potential $V$ mapped to a sine-Gordon potential] as a function of the interaction strength in the wire $K_{\mathrm{w}}$ [cf. Eq. (4)].

In this Letter, we fill this gap by formulating a lowenergy theory for a quantum wire in contact with arbitrary Ohmic-class leads, and show how these dramatically modify the low-energy properties of the wire. To highlight the consequences of this open system interplay, we analyze Kane-Fisher's impurity problem as a concrete setup. We show that while a super-Ohmic environment (with fast fluctuations) localizes the particles akin to a Zeno effect, sub-Ohmic noise (slow fluctuations) overwhelms the lowenergy properties of the TLL. The impurity, then, engenders the TLL-environment competition with a nonmonotonous renormalization group (RG) flow, leading ultimately to a conducting-to-insulating-like transition in the TLL as a function of the noise statistics in the environment. Furthermore, considering realistic finite-size one-dimensional (1D) systems, we predict that the nonmonotonous flow implies unusual temperature-dependent scaling of the conductance coming out from the TLL-environment competition.

Setup and microscopic model. We consider a system of interacting spinless electrons confined in a single-channel 1D wire of length $L$ that is adiabatically connected to metallic leads [see Fig. 1(a)]. The Hamiltonian of the wire reads

$$
H_{\mathrm{w}}=\int_{x}\left[\sum_{\eta=\mathrm{L}, \mathrm{R}}\left\{i \alpha_{\eta} \nu_{\mathrm{F}} \Psi_{\eta}^{\dagger}(x) \partial_{x} \Psi_{\eta}(x)+U \rho_{\eta}(x) \rho_{\eta}(x)\right\}\right],
$$

with $\int_{x}=\int_{-L / 2}^{L / 2} d x$, where the first term represents the kinetic energy of electrons with a linearized dispersion $\epsilon_{k}=$ $\alpha_{\eta} v_{\mathrm{F}} k, v_{\mathrm{F}}$ the electron velocity, and $\alpha_{\mathrm{L}}=1\left(\alpha_{\mathrm{R}}=-1\right)$ corresponds to the left- (right-)moving electrons with fermionic field operators $\Psi_{\mathrm{L}}\left(\Psi_{\mathrm{R}}\right)$. The second term describes local electron-electron interactions inside the wire via (normalordered) density operators $\rho_{\eta}(x)=: \Psi_{\eta}^{\dagger}(x) \Psi_{\eta}(x)$ : with a constant magnitude $U$.

Many-body interactions in the wire modify the relevant quasiparticles profoundly, resulting in the emergence of collective bosonic excitations [1,3,4]. Using bosonization, we can write the fermionic fields as $\Psi_{\eta}=\sqrt{\Lambda /(2 \pi)} \hat{F}_{\eta} \exp \left[i k_{\mathrm{F}} x+i \phi_{\eta}(x)\right]$, where $\Lambda$ is an ultraviolet cutoff, $\hat{F}_{\eta}$ the Klein factor, and $\phi_{\eta}$ represents bosonic fields with commutation relations, $\left[\phi_{\eta}(x), \phi_{\eta^{\prime}}(y)\right]=$ $-i \pi \alpha_{\eta} \delta_{\eta, \eta^{\prime}} \operatorname{sgn}(x-y)$. The density operator in terms of these bosonic fields reads $\rho_{\eta}(x)=\partial_{x} \phi_{\eta}(x) /(2 \pi)$. Thereby, the bosonized Hamiltonian of the interacting wire takes the form $H_{\mathrm{w}}=\frac{v}{4 \pi} \int_{x}\left\{\frac{1}{K_{\mathrm{w}}}\left(\partial_{x} \varphi\right)^{2}+K_{\mathrm{w}}\left(\partial_{x} \theta\right)^{2}\right\}$, where $\varphi(x, t), \theta(x, t)=(1 / \sqrt{2})\left[\phi_{\mathrm{L}}(x, t) \pm \phi_{\mathrm{R}}(x, t)\right]$ satisfy the commutation relation $[\varphi(x), \theta(y)]=i \pi \operatorname{sgn}(x-y)$, $v=v_{\mathrm{F}} / K_{\mathrm{w}}$, and $K_{\mathrm{w}}=1 / \sqrt{1+U /\left(\pi v_{\mathrm{F}}\right)}$ is the so-called Luttinger liquid parameter, with $K_{\mathrm{w}}=1$ referring to a noninteracting wire, and $K_{\mathrm{w}}<1\left(K_{\mathrm{w}}>1\right)$ indicating repulsive (attractive) interactions.

The wire is connected to electronic leads, which we introduce by imposing appropriate boundary conditions $\partial_{t} \phi_{\eta}(x=$ $\pm L / 2)=2 \pi J_{\eta}(t)$, where $J_{\eta}(\omega)$ is the current operator in the leads. Assuming an equilibrium situation $\left\langle J_{\eta}(\omega)\right\rangle=0$, where $\langle\cdots\rangle$ denotes thermal averaging with respect to the leads, the effect of the boundaries enters the correlation functions of the wire via the noise-power spectrum $S(\omega)=\left\langle J_{\eta}(\omega) J_{\eta}(-\omega)\right\rangle$ [76,83-85]. We consider an Ohmic-class noise-power spectrum

$$
S(\omega)=\omega\left|\frac{\omega}{\omega_{c}}\right|^{s-1} e^{-\left|\omega / \omega_{c}\right|}\left[1+n_{b}(\beta \omega)\right],
$$

where $\omega_{c}$ is the characteristic energy scale of the environment, indicating the exponential suppression of current-current correlations for $\omega \gg \omega_{c}$. The parameter $s \in(0,2)$ distinguishes between different cases, i.e., $s=1$ describes an Ohmic lead, whereas $s<1(s>1)$ corresponds to the sub- (super-) Ohmic case. The noise power exhibits a bosonic distribution $n_{b}(\beta \omega)=1 /[\exp (\beta \omega)-1]$ at inverse temperature $\beta$.

To realize an Ohmic environment, it suffices to consider free fermions with a well-defined Fermi-Dirac distribution. On the other hand, non-Ohmic environments with $s \neq 1$ can be realized, for example, by electron-phonon coupling in the leads $(s>1)$ [80], or by complex $R C$ circuit architectures $(s<1)$ [82]. In Fig. 1(b), we plot the frequency dependence of the noise spectrum for these three cases. Comparing to the Ohmic case, the current-current fluctuations in the sub(super)-Ohmic leads are more dominant at lower (higher) frequencies, i.e., environmental fluctuations are slower (faster). Ohmic-class environments have been extensively studied in the framework of the spin-boson model $[82,83,86-88]$, revealing the profound influence of the environment fluctuations on the nature of the ground state, as well as on the dynamics of the system. In particular, it was shown that in the Ohmic case, there exists a critical dissipation that distinguishes between a localized phase and a delocalized one [see Fig. 1(c)]. In contrast, in the sub- (super)-Ohmic case, the system is 
argued to be localized (delocalized) independent of dissipation strength [83]. Analogously, in this Letter, we investigate the impact of such current fluctuations in the leads on the transport through a disordered interacting wire.

Environment-induced correlations. First, we consider the limit of $T=0$, and investigate the impact of the noise spectrum in the leads on the TLL physics. The corre- lations between bosonic excitations of TLL are entirely determined by the Hamiltonian (1) and the noise-power spectrum at the boundaries (2) [76,85]. In particular, the wire's bosonic greater Green's function defined as $\mathcal{G}_{\varphi \varphi}^{>, 0}\left(x, x^{\prime}, \omega\right) \equiv-i\left\langle\varphi(x, \omega) \varphi^{\dagger}\left(x^{\prime}, \omega\right)\right\rangle_{0}$, with $\langle\cdots\rangle_{0}$ referring here to the thermal average, is found to be $\mathcal{G}_{\varphi \varphi}^{>, 0}\left(x, x^{\prime}, \omega\right)=$ $-i S(\omega) F_{\varphi}\left(x, x^{\prime}, \omega\right) / \omega^{2}$, with

$$
F_{\varphi}\left(x, x^{\prime}, \omega\right)=2 \frac{\left(\frac{1}{K_{\mathrm{w}}^{2}}-1\right) \cos \left[\frac{\omega \tau_{L}\left(x+x^{\prime}\right)}{L}\right] \cos \left[\omega \tau_{L}\right]+\left(\frac{1}{K_{\mathrm{w}}^{2}}+1\right) \cos \left[\frac{\omega \tau_{L}\left(x-x^{\prime}\right)}{L}\right]}{\left(1+\frac{1}{K_{\mathrm{w}}^{2}}\right)^{2}-\left(1-\frac{1}{K_{\mathrm{w}}^{2}}\right)^{2} \cos ^{2}\left[\omega \tau_{L}\right]},
$$

the structure function of a many-body Fabry-Pérot interferometer that is formed due to the presence of the leads reflecting the bosonic excitations at the boundaries [76,85]. Detailed balance holds $\mathcal{G}_{\varphi \varphi}^{<, 0}\left(x, x^{\prime}, \omega\right) \equiv$ $-i\left\langle\varphi^{\dagger}\left(x^{\prime}, \omega\right) \varphi(x, \omega)\right\rangle_{0}=e^{-\beta \omega} \mathcal{G}_{\varphi \varphi}^{>, 0}\left(x, x^{\prime}, \omega\right)$, as expected for bosons in thermal equilibrium [89]. The finite length of the wire introduces a characteristic timescale to the system, namely, the time of flight for the collective excitations $\tau_{\mathrm{L}}=$ $L K_{\mathrm{w}} / v_{\mathrm{F}}$ to cross the wire. At high frequencies, $\omega \tau_{\mathrm{L}} \gg 1$, the system acts similarly to the infinite wire [90], whereas at small frequencies, $\omega \tau_{\mathrm{L}} \ll 1, F_{\varphi}\left(x, x^{\prime}, \omega\right) \approx 1$ such that the physics of the interacting wire is washed out, and the system response is dominated by the environment-cf. Ref. [42] for the Ohmic environment case [91].

An impurity in the wire at zero temperature. To reveal the consequences of the environment-induced correlations, we consider a backscattering impurity at $x=x_{0}$, leading to an additional Hamiltonian term, $H_{b}=V_{0}\left[\Psi_{\mathrm{L}}^{\dagger}\left(x_{0}\right) \Psi_{\mathrm{R}}\left(x_{0}\right)+\right.$ H.c. $]$. The action of the bosonized system at all positions $x \neq x_{0}$ is quadratic and can be therefore integrated out, resulting in the following (local sine-Gordon) action in imaginary-time path-integral formalism

$$
\begin{aligned}
\mathcal{A}= & \int_{0}^{\beta} d \tau \varphi^{\dagger}(\tau)\left[\mathcal{G}_{\varphi \varphi}^{0}(\tau)\right]^{-1} \varphi(\tau) \\
& +V_{0} \int_{0}^{\beta} d \tau \cos [\sqrt{4 \pi} \varphi(\tau)],
\end{aligned}
$$

where $\mathcal{G}_{\varphi \varphi}^{0}(\tau)=\int_{-\infty}^{\infty} d \omega \mathcal{G}_{\varphi \varphi}^{0}(i \omega) e^{i \omega \tau}$, with $\quad \mathcal{G}_{\varphi \varphi}^{0}(i \omega)=$ $\int \frac{d \omega^{\prime}}{2 \pi i} \frac{\mathcal{G}_{\varphi \varphi}^{0,>}\left(\omega^{\prime}\right)-\mathcal{G}_{\varphi \varphi}^{0,<}\left(\omega^{\prime}\right)}{i \omega-\omega^{\prime}}$ the imaginary-time (Matsubara) Green's function of the clean wire at $x=x_{0}$. Without loss of generality, we assume $x_{0}=0$ [92]. The action (4) and its corresponding correlation functions describe the physics found in a variety of systems, including Brownian motion of a quantum mechanical particle in a periodic potential [93], as well as in the dissipative two-level system $[83,86,94,95]$; our specific wire-environment competition manifests through the explicit functional form of $\mathcal{G}_{\varphi \varphi}^{0}$.

The noise spectrum of Ohmic leads scales linearly at low energies, $S(\omega) \sim \omega$, and we commonly observe $\mathcal{G}_{\varphi \varphi}^{0}(i \omega)=$ $K_{\mathrm{w}} /|2 \omega|$. More generally, however, we can always define a similar structure $\mathcal{G}_{\varphi \varphi}^{0}(i \omega)=K(\omega) /|2 \omega|$ with an energydependent Luttinger parameter $K(\omega)$ incorporating the finite length of the wire, and the fluctuations from the leads. At low frequencies $\omega \ll \omega_{c}$, we have [90]

$$
K(\omega) \approx \frac{K_{\mathrm{w}}}{\sin (\pi s) / 2}\left|\frac{\omega}{\omega_{c}}\right|^{s-1} \frac{1+K_{\mathrm{w}}+\left(1-K_{\mathrm{w}}\right) e^{-\tau_{\mathrm{L}}|\omega|}}{1+K_{\mathrm{w}}-\left(1-K_{\mathrm{w}}\right) e^{-\tau_{\mathrm{L}}|\omega|}},
$$

which is plotted in Fig. 2(a) for the long-wire limit $\omega \gg 1 / \tau_{L}$. Equation (5) is one of the main results of this work, showcasing how the presence of leads, at the wire's ends, modifies the effective strength of the interparticle interactions in the wire at different frequencies. In particular, in the sub-Ohmic case (slow environmental fluctuations), at sufficiently low frequencies, the effective interactions in the wire appear attractive $[K(\omega)>1]$, while for the super-Ohmic case, the interactions become effectively repulsive $[K(\omega)<1]$.

We employ a perturbative RG approach (due to the presence of infrared divergences [82]), in which we integrate out high-energy fields and map the system (4) to itself, but with a smaller ultraviolet cutoff $\Lambda^{\prime}=\Lambda(1-d l)$, i.e., $d l=d \Lambda / \Lambda$ [18]. As a result, a renormalized scattering potential $V(\Lambda)$ (up

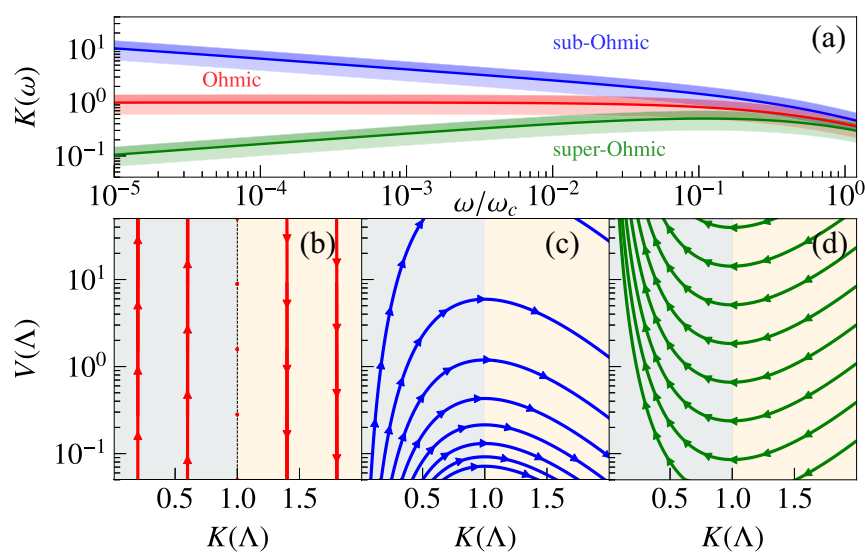

FIG. 2. Scaling and RG flow of the scattering potential $V(\omega)$ and effective Luttinger parameter $K(\omega)$ at zero temperature [cf. Eqs. (4)-(6)]. (a) The frequency dependence of $K(\omega)$ for $\omega \gg 1 / \tau_{L}$ for a range of interaction strengths in the wire $K_{\mathrm{w}} \in[0.6,1.4]$ and different Ohmic-class cases, $s=1.2,1.0,0.8$. The solid lines depict the noninteracting case $K_{\mathrm{w}}=1$, and the lighter (darker) shades mark $K_{\mathrm{w}}<1\left(K_{\mathrm{w}}>1\right)$. (b)-(d) The flow diagram (along the direction of the arrows) of the scattering potential vs effective interaction as we change the cutoff energy scale $\Lambda$ from $\left[\infty, \Lambda_{f}\right]$, with $\Lambda_{f}>1 / \tau_{L}$ for (b) Ohmic ( $s=1.0)$, (c) sub-Ohmic ( $s=0.8$ ), and (d) super-Ohmic $(s=1.2)$ cases. 
to $d l^{2}$ ) obeys the flow equation

$$
\frac{d V}{d l}=V[1-K(\Lambda)]
$$

Note that due to the noisy leads, the flow involves also the renormalization of $K(\omega)$. The numerical solution of the flow equation for the infinite wire limit is shown in Fig. 2, whereas the impact of the finite wire is detailed in the Supplemental Material [90]. For the Ohmic case $s=1$ [Fig. 2(b)], standard Kane-Fisher physics [18] is observed, where the wire's Luttinger parameter plays a decisive role, i.e., for $K_{\mathrm{W}}<1\left(K_{\mathrm{w}}>\right.$ $1)$ the fixed point of the RG, for $\Lambda \rightarrow 0$, is $V \rightarrow \infty(V \rightarrow 0)$ for the insulating (metallic) case. The quantum critical point is at $K_{\mathrm{W}}=1$, corresponding to noninteracting electrons [see Fig. 1(d)].

Considering sub-Ohmic environment noise [see Fig. 2(c)], the low-frequency noise induces an effective $K(\omega)$ that increases with $\Lambda$. Therefore, starting from repulsive interactions in the wire, the renormalized scattering potential exhibits a nonmonotonic behavior. Specifically, defining $\Lambda^{*}$ such that $K\left(\Lambda^{*}\right)=1$, we observe that for $\Lambda>\Lambda^{*}$, the backscattering potential increases and transport through the wire is suppressed, while for $\Lambda<\Lambda^{*}$, the potential decreases and transport is unaffected by the impurity. The transition point $\Lambda^{*}$ strongly depends on the wire's bare Luttinger liquid parameter $K_{\mathrm{w}}$ [cf. Eq. (5)]. Crucially, regardless of the specific initial microscopic parameters of the wire, the fixed point of the flow is $K \rightarrow \infty, V \rightarrow 0$. We compare the sub-Ohmic case with the super-Ohmic one, where the flow lines show an opposite trend [see Fig. 2(d)]. In this case, the effective $K(\omega)$ is reduced and the fixed point is realized at $K \rightarrow 0, V \rightarrow \infty$.

Our perturbative RG analysis assumes an initially small scattering impurity potential. In the opposite limit of a strong impurity, we can formulate the problem in a dual representation with $\mathcal{G}_{\theta \theta}(i \omega)=1 /[2 \tilde{K}(\omega)|\omega|]$ [90], with $\tilde{K}(\omega)$ defined by changing $(1-s) \rightarrow(s-1)$ in Eq. (5). Hence, the sub-Ohmic environment acts as a super-Ohmic one, and vice versa [96]. As a result, tunneling $t$ across the barrier satisfies the $\mathrm{RG}$ equation $d t / d l=t\{1-[1 / \tilde{K}(\Lambda)]\}$. We conclude that the metal-to-insulator quantum phase transition that occurs for the Ohmic environment at the critical point, $K_{\mathrm{w}}=1$, is replaced for non-Ohmic environments by a smooth crossover with a characteristic energy scale $\Lambda^{*}$ that depends on the interaction strength in the wire.

Conductance at finite temperatures. We study ac conductance through the wire, and consider an external probe in the form of a potential $U(x, t)=U(x) \cos (\omega t)$, leading to an additional term in the Hamiltonian, $\delta H=\sum_{\eta} \int d x \rho_{\eta}(x) U(x)$. Within linear response theory [71,72,90,97], the ac conductance reads $G(\omega)=-\left(e^{2} / h\right) 2 i \omega \mathcal{G}_{\varphi \varphi}^{\mathrm{R}}(\omega)$, with the retarded plasmonic Green's function in the presence of the impurity $\mathcal{G}_{\varphi \varphi}^{\mathrm{R}}(\omega)=\left.\int \mathcal{D}[\varphi] \varphi(\omega) \varphi^{\dagger}(\omega) e^{-\mathcal{A}}\right|_{\omega+i 0^{+}}=$ $\left[\left(\mathcal{G}_{\varphi \varphi}^{0, \mathrm{R}}\right)^{-1}(\omega)-\Sigma^{\mathrm{R}}(\omega)\right]^{-1}$, where the self-energy can be obtained by expanding the partition function corresponding to the action (4) [90], which up to $V_{0}^{2}$ reads

$$
\Sigma(i \omega)=i V_{0}^{2} \int_{0}^{\beta} d \tau\left[1-e^{i \omega \tau}\right] e^{E(\tau)}
$$

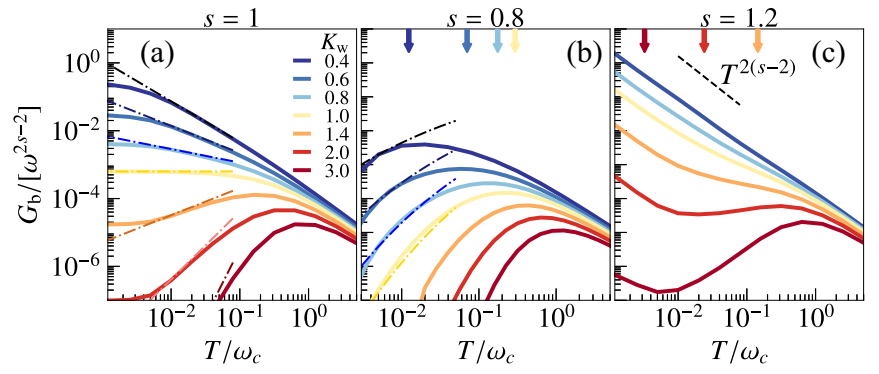

FIG. 3. Temperature dependence of the impurity-induced correction to conductance through a finite-length wire $\left[L=100 v_{\mathrm{F}} /\left(K_{\mathrm{w}} \omega_{c}\right)\right]$ for different interaction strengths $\left(K_{\mathrm{w}}\right)$ [cf. Eq. (7)]. (a) Ohmic, (b) sub-Ohmic $(s=0.8)$, and (c) super-Ohmic case $(s=1.2)$. The dashed lines show in (a) the expected power-law dependence $G_{b} \propto\left(T / \omega_{c}\right)^{2 K_{\mathrm{w}}-2}$, (b) the exponential dependence $G_{b} \propto$ $\exp \left[-\alpha_{s}\left(T / \omega_{c}\right)^{s-1}\right]$, and (c) $G_{b} \propto\left(T / \omega_{c}\right)^{2 s-4}$ [90]. The arrows in (b) and (c) mark $\Lambda^{*}$, where $K\left(\Lambda^{*}\right)=1$.

with

$$
E(\tau)=\int_{0}^{\infty} \frac{d \omega}{2 \pi}\left\{\frac{1-\cosh (\omega \tau)}{\tanh (\beta \omega / 2)}+\sinh (\omega \tau)\right\} \mathcal{G}_{\varphi \varphi}^{0}(\omega)
$$

Thereby, the ac conductance can be written as $G(\omega)=$ $\left(e^{2} / h\right)\left[G_{0}-G_{b}\right]$, where $G_{0}=-2 i \omega \mathcal{G}_{\varphi \varphi}^{\mathrm{R}, 0}(\omega)$ corresponds to the conductance through a clean wire, and $G_{b} \equiv$ $-2 i \omega \mathcal{G}_{\varphi \varphi}^{\mathrm{R}, 0} \Sigma^{\mathrm{R}}(\omega) \mathcal{G}_{\varphi \varphi}^{\mathrm{R}, 0}$ represents the correction to the conductance due to the presence of the impurity. In the dc limit, $\lim _{\omega \rightarrow 0} G_{0}(\omega)=\left(\omega / \omega_{c}\right)^{s-1}$, which is independent of temperature and interaction strength inside the wire. For the Ohmic case, $\lim _{\omega \rightarrow 0} G_{0}(\omega)=1$, for sub-Ohmic this limit diverges, and in the super-Ohmic case it vanishes.

In the following, we focus on the temperature-dependence of the correction $G_{b}$ (see Fig. 3). The temperature mimics the RG flow of the renormalized scattering potential (cf. Fig. 2): (i) In the Ohmic case [Fig. 3(a)], for $1 / \tau_{\mathrm{L}} \ll T \ll \omega_{c}$, we obtain a power-law temperature dependence of the form $G_{b} \propto$ $\left(T / \omega_{c}\right)^{2 K_{\mathrm{w}}-2}$. Such a power law is characteristic for critical scaling close to a quantum phase transition [90]. Specifically, for a repulsive interaction, $G_{b}$ grows with decreasing temperature, while it gets suppressed for attractive interactions. For a noninteracting wire, $G_{b}$ is independent of temperature. Interestingly, at small temperatures $\left(T \tau_{\mathrm{L}} \ll 1\right)$, we observe a temperature-independent behavior for all values of $K_{\mathrm{w}}$, corresponding to the cutoff of the critical scaling by the finite length of the wire: (ii) For the sub- and super-Ohmic case [Figs. 3(b) and 3(c), respectively], we observe a characteristic energy scale $\Lambda^{*}$ above which the system qualitatively behaves as in the Ohmic case, i.e., with a power-law decrease governed by the noise scaling with $\omega_{c}$. However, at $T \ll \Lambda^{*}$, for the sub-Ohmic case, we obtain an exponential suppression with exponents depending on the interaction strength as $G_{b} \propto \exp \left[-K_{\mathrm{w}} \alpha_{s}\left(T / \omega_{c}\right)^{s-1}\right]$. In contrast, in the super-Ohmic case for $T \ll \Lambda^{*}$, the $G_{b}$ grows with decreasing temperature in a power-law fashion with an exponent that is entirely independent of the interaction strength in the wire $T^{2 s-4}$. The finite length effect at low temperatures is washed away by the noisy environment.

Conclusion. We show that the specifics of charge fluctuation at the boundaries of an interacting wire can modify 
the transport through a dirty Luttinger liquid beyond the Kane-Fisher description. We further outline the physical implications of the wire-environment competition for realistic transport measurements in a wide variety of systems. Specifically, at low temperatures, the impurity-induced correction to conductance (i) follows the result of Kane-Fisher [18], and critically scales with an interaction-dependent power law up to a finite length cutoff in the Ohmic case, (ii) gets washed out in the sub-Ohmic case due to the dominant role of slow (viscous) fluctuations in the environment, and (iii) is effectively amplified as the fast charge fluctuations (super-Ohmic) at the boundary of the wire act similarly to a Zeno effect [83]. Our results highlight bath engineering as a tool to design different phases of matter, without the drawback of inducing dissipation. Furthermore, we pave the way toward analyzing, e.g., the interplay between non-Ohmic reservoirs and a macroscopic number of in-wire impurities, where another type of metal-to-insulator quantum phase transition is predicted to occur [3], or the investigation of bath-induced stabilization protocols for exotic excitations, such as Majorana fermions. Further interesting extensions of our work involve the inclusion of the spin, considering the range of interactions [79,98], and investigating the backaction of the quantum wire on the environment.

Acknowledgments. A.K., A.S., and O.Z. acknowledge financial support from the Swiss National Science Foundation. A.C. acknowledges support by the funding from the $\mathrm{Eu}-$ ropean Research Council (ERC) under the Horizon 2020 research and innovation program, Grant Agreement No. 647434 (DOQS).
[1] J. von Delft and H. Schoeller, Bosonization for beginners refermionization for experts, Ann. Phys. 7, 225 (1998).

[2] F. D. M. Haldane, "Luttinger liquid theory" of one-dimensional quantum fluids. I. Properties of the Luttinger model and their extension to the general 1D interacting spinless Fermi gas, J. Phys. C 14, 2585 (1981).

[3] T. Giamarchi, Quantum Physics in One Dimension, International Series of Monographs (Clarendon Press, Oxford, UK, 2004).

[4] A. O. Gogolin, A. A. Nersesyan, and A. M. Tsvelik, Bosonization and Strongly Correlated Systems (Cambridge University Press, Cambridge, UK, 2004).

[5] M. A. Cazalilla, R. Citro, T. Giamarchi, E. Orignac, and M. Rigol, One dimensional bosons: From condensed matter systems to ultracold gases, Rev. Mod. Phys. 83, 1405 (2011).

[6] T. Giamarchi, Some experimental tests of Tomonaga-Luttinger liquids, Int. J. Mod. Phys. B 26, 1244004 (2012).

[7] O. M. Auslaender, A. Yacoby, R. de Picciotto, K. W. Baldwin, L. N. Pfeiffer, and K. W. West, Tunneling spectroscopy of the elementary excitations in a one-dimensional wire, Science $\mathbf{2 9 5}$, 825 (2002).

[8] Y. Jompol, C. J. B. Ford, J. P. Griffiths, I. Farrer, G. A. C. Jones, D. Anderson, D. A. Ritchie, T. W. Silk, and A. J. Schofield, Probing spin-charge separation in a Tomonaga-Luttinger liquid, Science 325, 597 (2009).

[9] E. Bocquillon, V. Freulon, J.-M. Berroir, P. Degiovanni, B. Plaçais, A. Cavanna, Y. Jin, and G. Fève, Separation of neutral and charge modes in one-dimensional chiral edge channels, Nat. Commun. 4, 1839 (2013).

[10] M. Hashisaka, N. Hiyama, T. Akiho, K. Muraki, and T. Fujisawa, Waveform measurement of charge- and spin-density wavepackets in a chiral Tomonaga-Luttinger liquid, Nat. Phys. 13, 559 (2017).

[11] H. Steinberg, G. Barak, A. Yacoby, L. N. Pfeiffer, K. W. West, B. I. Halperin, and K. Le Hur, Charge fractionalization in quantum wires, Nat. Phys. 4, 116 (2008).

[12] M. G. Prokudina, S. Ludwig, V. Pellegrini, L. Sorba, G. Biasiol, and V. S. Khrapai, Tunable Nonequilibrium Luttinger Liquid based on Counterpropagating Edge Channels, Phys. Rev. Lett. 112, 216402 (2014).
[13] H. Kamata, N. Kumada, M. Hashisaka, K. Muraki, and T. Fujisawa, Fractionalized wave packets from an artificial Tomonaga-Luttinger liquid, Nat. Nanotechnol. 9, 177 (2014).

[14] V. Freulon, A. Marguerite, J.-M. Berroir, B. Plaçais, A. Cavanna, Y. Jin, and G. Fève, Hong-Ou-Mandel experiment for temporal investigation of single-electron fractionalization, Nat. Commun. 6, 6854 (2015).

[15] K. A. Matveev and L. I. Glazman, Coulomb Blockade of Tunneling into a Quasi-One-Dimensional Wire, Phys. Rev. Lett. 70, 990 (1993).

[16] M. Bockrath, D. H. Cobden, J. Lu, A. G. Rinzler, R. E. Smalley, L. Balents, and P. L. McEuen, Luttinger-liquid behaviour in carbon nanotubes, Nature (London) 397, 598 (1999).

[17] Z. Yao, H. W. Ch. Postma, L. Balents, and C. Dekker, Carbon nanotube intramolecular junctions, Nature (London) 402, 273 (1999).

[18] C. L. Kane and M. P. A. Fisher, Transport in a One-Channel Luttinger Liquid, Phys. Rev. Lett. 68, 1220 (1992).

[19] C. L. Kane and M. P. A. Fisher, Transmission through barriers and resonant tunneling in an interacting one-dimensional electron gas, Phys. Rev. B 46, 15233 (1992).

[20] A. Anthore, D. M. Kennes, E. Boulat, S. Andergassen, F. Pierre, and V. Meden, Universality at work - the local sine-Gordon model, lattice fermions, and quantum circuits, Eur. Phys. J.: Spec. Top. 229, 663 (2020).

[21] K. A. Matveev, D. Yue, and L. I. Glazman, Tunneling in OneDimensional Non-Luttinger Electron Liquid, Phys. Rev. Lett. 71, 3351 (1993).

[22] S. Qin, M. Fabrizio, and L. Yu, Impurity in a Luttinger liquid: A numerical study of the finite-size energy spectrum and of the orthogonality catastrophe exponent, Phys. Rev. B 54, R9643 (1996).

[23] V. Meden, P. Schmitteckert, and N. Shannon, Orthogonality catastrophe in a one-dimensional system of correlated electrons, Phys. Rev. B 57, 8878 (1998).

[24] D. M. Kennes, M. J. Schmidt, D. Hübscher, and V. Meden, Renormalization group flows in one-dimensional lattice models: Impurity scaling, umklapp scattering, and the orthogonality catastrophe, Phys. Rev. B 90, 155129 (2014).

[25] A. Anthore, Z. Iftikhar, E. Boulat, F. D. Parmentier, A. Cavanna, A. Ouerghi, U. Gennser, and F. Pierre, Circuit 
Quantum Simulation of a Tomonaga-Luttinger Liquid with an Impurity, Phys. Rev. X 8, 031075 (2018).

[26] J. Cao, Q. Wang, and H. Dai, Electron transport in very clean, as-grown suspended carbon nanotubes, Nat. Mater. 4, 745 (2005).

[27] X. G. Wen, Chiral Luttinger liquid and the edge excitations in the fractional quantum Hall states, Phys. Rev. B 41, 12838 (1990).

[28] A. M. Chang, Chiral Luttinger liquids at the fractional quantum Hall edge, Rev. Mod. Phys. 75, 1449 (2003).

[29] B. Yang, Y.-Y. Chen, Y.-G. Zheng, H. Sun, H.-N. Dai, X.-W. Guan, Z.-S. Yuan, and J.-W. Pan, Quantum Criticality and the Tomonaga-Luttinger Liquid in One-Dimensional Bose Gases, Phys. Rev. Lett. 119, 165701 (2017).

[30] M. Lebrat, P. Grišins, D. Husmann, S. Häusler, L. Corman, T. Giamarchi, J.-P. Brantut, and T. Esslinger, Band and Correlated Insulators of Cold Fermions in a Mesoscopic Lattice, Phys. Rev. X 8, 011053 (2018).

[31] T. L. Yang, P. Grišins, Y. T. Chang, Z. H. Zhao, C. Y. Shih, T. Giamarchi, and R. G. Hulet, Measurement of the Dynamical Structure Factor of a 1D Interacting Fermi Gas, Phys. Rev. Lett. 121, 103001 (2018).

[32] B. Lake, D. A. Tennant, C. D. Frost, and S. E. Nagler, Quantum criticality and universal scaling of a quantum antiferromagnet, Nat. Mater. 4, 329 (2005).

[33] E. Dagotto, Experiments on ladders reveal a complex interplay between a spin-gapped normal state and superconductivity, Rep. Prog. Phys. 62, 1525 (1999).

[34] M. Klanjšek, H. Mayaffre, C. Berthier, M. Horvatić, B. Chiari, O. Piovesana, P. Bouillot, C. Kollath, E. Orignac, R. Citro, and T. Giamarchi, Controlling Luttinger Liquid Physics in Spin Ladders under a Magnetic Field, Phys. Rev. Lett. 101, 137207 (2008).

[35] K. Itoh, R. Nakazawa, T. Ota, M. Hashisaka, K. Muraki, and T. Fujisawa, Signatures of a Nonthermal Metastable State in Copropagating Quantum Hall Edge Channels, Phys. Rev. Lett. 120, 197701 (2018)

[36] G. Yang, Y. Shao, J. Niu, X. Ma, C. Lu, W. Wei, X. Chuai, J. Wang, J. Cao, H. Huang, G. Xu, X. Shi, Z. Ji, N. Lu, D. Geng, J. Qi, Y. Cao, Z. Liu, L. Liu, Y. Huang et al., Possible Luttinger liquid behavior of edge transport in monolayer transition metal dichalcogenide crystals, Nat. Commun. 11, 659 (2020).

[37] I. Bloch, J. Dalibard, and W. Zwerger, Many-body physics with ultracold gases, Rev. Mod. Phys. 80, 885 (2008).

[38] I. Bloch, J. Dalibard, and S. Nascimbène, Quantum simulations with ultracold quantum gases, Nat. Phys. 8, 267 (2012).

[39] I. M. Georgescu, S. Ashhab, and F. Nori, Quantum simulation, Rev. Mod. Phys. 86, 153 (2014).

[40] T. Langen, R. Geiger, and J. Schmiedmayer, Ultracold atoms out of equilibrium, Annu. Rev. Condens. Matter Phys. 6, 201 (2015).

[41] S. Krinner, T. Esslinger, and J.-P. Brantut, Two-terminal transport measurements with cold atoms, J. Phys.: Condens. Matter 29, 343003 (2017).

[42] I. Safi and H. Saleur, One-Channel Conductor in an Ohmic Environment: Mapping to a Tomonaga-Luttinger Liquid and Full Counting Statistics, Phys. Rev. Lett. 93, 126602 (2004).

[43] S. Jezouin, M. Albert, F. D. Parmentier, A. Anthore, U. Gennser, A. Cavanna, I. Safi, and F. Pierre,
Tomonaga-Luttinger physics in electronic quantum circuits, Nat. Commun. 4, 1802 (2013).

[44] J. Eisert, M. Friesdorf, and C. Gogolin, Quantum many-body systems out of equilibrium, Nat. Phys. 11, 124 (2015).

[45] A. H. Castro Neto, C. de C. Chamon, and C. Nayak, Open Luttinger Liquids, Phys. Rev. Lett. 79, 4629 (1997).

[46] M. A. Cazalilla, F. Sols, and F. Guinea, Dissipation-Driven Quantum Phase Transitions in a Tomonaga-Luttinger Liquid Electrostatically Coupled to a Metallic Gate, Phys. Rev. Lett. 97, 076401 (2006).

[47] Z. Ristivojevic and T. Nattermann, Transport in a Dissipative Luttinger Liquid, Phys. Rev. Lett. 101, 016405 (2008).

[48] A. Altland, Y. Gefen, and B. Rosenow, Incoherent Scatterer in a Luttinger Liquid: A New Paradigmatic Limit, Phys. Rev. Lett. 108, 136401 (2012).

[49] M. Soriente, T. Donner, R. Chitra, and O. Zilberberg, Dissipation-Induced Anomalous Multicritical Phenomena, Phys. Rev. Lett. 120, 183603 (2018).

[50] F. Tonielli, R. Fazio, S. Diehl, and J. Marino, Orthogonality Catastrophe in Dissipative Quantum Many-Body Systems, Phys. Rev. Lett. 122, 040604 (2019).

[51] N. Dogra, M. Landini, K. Kroeger, L. Hruby, T. Donner, and T. Esslinger, Dissipation-induced structural instability and chiral dynamics in a quantum gas, Science 366, 1496 (2019).

[52] M. Soriente, R. Chitra, and O. Zilberberg, Distinguishing phases using the dynamical response of driven-dissipative lightmatter systems, Phys. Rev. A 101, 023823 (2020).

[53] M. S. Ferguson, L. C. Camenzind, C. Müller, D. E. F. Biesinger, C. P. Scheller, B. Braunecker, D. M. Zumbühl, and O. Zilberberg, Quantum measurement induces a many-body transition, arXiv:2010.04635.

[54] M. Soriente, T. L. Heugel, K. Arimitsu, R. Chitra, and O. Zilberberg, Distinctive class of dissipation-induced phase transitions and their universal characteristics, Phys. Rev. Research 3, 023100 (2021).

[55] G. Barontini, R. Labouvie, F. Stubenrauch, A. Vogler, V. Guarrera, and H. Ott, Controlling the Dynamics of an Open Many-Body Quantum System with Localized Dissipation, Phys. Rev. Lett. 110, 035302 (2013).

[56] R. Labouvie, B. Santra, S. Heun, and H. Ott, Bistability in a Driven-Dissipative Superfluid, Phys. Rev. Lett. 116, 235302 (2016).

[57] H. Fröml, A. Chiocchetta, C. Kollath, and S. Diehl, FluctuationInduced Quantum Zeno Effect, Phys. Rev. Lett. 122, 040402 (2019).

[58] H. Fröml, C. Muckel, C. Kollath, A. Chiocchetta, and S. Diehl, Ultracold quantum wires with localized losses: Many-body quantum Zeno effect, Phys. Rev. B 101, 144301 (2020).

[59] D. A. Zezyulin, V. V. Konotop, G. Barontini, and H. Ott, Macroscopic Zeno Effect and Stationary Flows in Nonlinear Waveguides with Localized Dissipation, Phys. Rev. Lett. 109, 020405 (2012).

[60] B. Buča, C. Booker, M. Medenjak, and D. Jaksch, Bethe ansatz approach for dissipation: Exact solutions of quantum many-body dynamics under loss, New J. Phys. 22, 123040 (2020).

[61] N. Syassen, D. M. Bauer, M. Lettner, T. Volz, D. Dietze, J. J. García-Ripoll, J. I. Cirac, G. Rempe, and S. Dürr, Strong dissipation inhibits losses and induces correlations in cold molecular gases, Science 320, 1329 (2008). 
[62] J. J. García-Ripoll, S. Dürr, N. Syassen, D. M. Bauer, M. Lettner, G. Rempe, and J. I. Cirac, Dissipation-induced hardcore boson gas in an optical lattice, New J. Phys. 11, 013053 (2009).

[63] M. Nakagawa, N. Kawakami, and M. Ueda, Exact Liouvillian Spectrum of a One-Dimensional Dissipative Hubbard Model, Phys. Rev. Lett. 126, 110404 (2021).

[64] D. B. Gutman, Y. Gefen, and A. D. Mirlin, Nonequilibrium Luttinger Liquid: Zero-Bias Anomaly and Dephasing, Phys. Rev. Lett. 101, 126802 (2008).

[65] D. B. Gutman, Y. Gefen, and A. D. Mirlin, Tunneling spectroscopy of Luttinger-liquid structures far from equilibrium, Phys. Rev. B 80, 045106 (2009).

[66] D. B. Gutman, Y. Gefen, and A. D. Mirlin, Bosonization of one-dimensional fermions out of equilibrium, Phys. Rev. B 81, 085436 (2010).

[67] S. Ngo Dinh, D. A. Bagrets, and A. D. Mirlin, Tunneling into a nonequilibrium Luttinger liquid with impurity, Phys. Rev. B 81, 081306(R) (2010).

[68] C. Karrasch, J. Rentrop, D. Schuricht, and V. Meden, LuttingerLiquid Universality in the Time Evolution after an Interaction Quench, Phys. Rev. Lett. 109, 126406 (2012).

[69] S. Tarucha, T. Honda, and T. Saku, Reduction of quantized conductance at low temperatures observed in 2 to $10 \mu \mathrm{m}$-long quantum wires, Solid State Commun. 94, 413 (1995).

[70] M. Ogata and H. Fukuyama, Collapse of Quantized Conductance in a Dirty Tomonaga-Luttinger Liquid, Phys. Rev. Lett. 73, 468 (1994).

[71] A. Furusaki and N. Nagaosa, Tunneling through a barrier in a Tomonaga-Luttinger liquid connected to reservoirs, Phys. Rev. B 54, R5239 (1996).

[72] D. L. Maslov, Transport through dirty Luttinger liquids connected to reservoirs, Phys. Rev. B 52, R14368(R) (1995).

[73] A. Furusaki and N. Nagaosa, Resonant tunneling in a Luttinger liquid, Phys. Rev. B 47, 3827 (1993).

[74] C. L. Kane, Transmission through barriers and resonant tunneling in a Luttinger liquid, Phys. B: Condens. Matter 189, 250 (1993).

[75] V. Meden, S. Andergassen, T. Enss, H. Schoeller, and K. Schönhammer, Fermionic renormalization group methods for transport through inhomogeneous Luttinger liquids, New J. Phys. 10, 045012 (2008).

[76] A. Štrkalj, M. S. Ferguson, T. M. R. Wolf, I. Levkivskyi, and O. Zilberberg, Tunneling into a Finite Luttinger Liquid Coupled to Noisy Capacitive Leads, Phys. Rev. Lett. 122, 126802 (2019).

[77] I. Safi and H. J. Schulz, Transport in an inhomogeneous interacting one-dimensional system, Phys. Rev. B 52, R17040 (1995).

[78] A. Yacoby, H. L. Stormer, N. S. Wingreen, L. N. Pfeiffer, K. W. Baldwin, and K. W. West, Nonuniversal Conductance Quantization in Quantum Wires, Phys. Rev. Lett. 77, 4612 (1996).

[79] M. Fabrizio, A. O. Gogolin, and S. Scheidl, Coulomb Effects in Transport Properties of Quantum Wires, Phys. Rev. Lett. 72, 2235 (1994).

[80] J. P. Sethna, Decay rates of tunneling centers coupled to phonons: An instanton approach, Phys. Rev. B 25, 5050 (1982).

[81] D. Husmann, S. Uchino, S. Krinner, M. Lebrat, T. Giamarchi, T. Esslinger, and J.-P. Brantut, Connecting strongly correlated superfluids by a quantum point contact, Science 350, 1498 (2015).

[82] U. Weiss, Quantum Dissipative Systems, Series in Modern Condensed Matter Physics (World Scientific, Singapore, 1999).

[83] A. J. Leggett, S. Chakravarty, A. T. Dorsey, M. P. A. Fisher, A. Garg, and W. Zwerger, Dynamics of the dissipative two-state system, Rev. Mod. Phys. 59, 1 (1987).

[84] M. Büttiker, Scattering theory of current and intensity noise correlations in conductors and wave guides, Phys. Rev. B 46, 12485 (1992).

[85] Y. V. Nazarov, A. A. Odintsov, and D. V. Averin, Tunnel density of states in a Luttinger constriction, Europhys. Lett. 37, 213 (1997).

[86] M. Vojta, N.-H. Tong, and R. Bulla, Quantum Phase Transitions in the Sub-Ohmic Spin-Boson Model: Failure of the Quantum-Classical Mapping, Phys. Rev. Lett. 94, 070604 (2005).

[87] F. B. Anders, R. Bulla, and M. Vojta, Equilibrium and Nonequilibrium Dynamics of the Sub-Ohmic Spin-Boson Model, Phys. Rev. Lett. 98, 210402 (2007).

[88] S. K. Kehrein, A. Mielke, and P. Neu, Flow equations for the spin-boson problem, Z. Phys. B 99, 269 (1995).

[89] H. Bruus and K. Flensberg, Many-Body Quantum Theory in Condensed Matter Physics, Oxford Graduate Texts (Oxford University Press, Oxford, UK, 2004).

[90] See Supplemental Material at http://link.aps.org/supplemental/ 10.1103/PhysRevResearch.3.L032013 for additional details on (1) bosonic Green's functions of a clean wire obtained from the equations of motion for bosonic fields, (2) the impact of Ohmicclass environment on the wire's correlation functions, (3) the renormalization of the scattering potential, and (4) conductance calculations through the wire.

[91] The case of interacting one-dimensional leads with a TLL parameter $K_{1} \neq 1$ can be further included within our framework by considering a leads' power spectrum $S(\omega)=K_{\mathrm{l}} \omega e^{-\left|\omega / \omega_{c}\right|}[1+$ $\left.n_{b}(\beta \omega)\right]$, as compared to Eq. (2).

[92] The generalization to $x_{0} \neq 0$ can be readily carried out in an analogous ways-see, for example, Ref. [71].

[93] M. P. A. Fisher and W. Zwerger, Quantum Brownian motion in a periodic potential, Phys. Rev. B 32, 6190 (1985).

[94] S. Florens, D. Venturelli, and R. Narayanan, Quantum phase transition in the spin boson model, Quantum Quenching, Annealing and Computation, edited by A. Kumar Chandra, A. Das, and B. K. Chakrabarti (Springer, Berlin, 2010), pp. 145-162.

[95] M. Vojta, Numerical renormalization group for the sub-Ohmic spin-boson model: A conspiracy of errors, Phys. Rev. B 85, 115113 (2012).

[96] M. Sassetti, H. Schomerus, and U. Weiss, Subdiffusive and superdiffusive quantum transport and generalized duality, Phys. Rev. B 53, R2914 (1996).

[97] M. P. A. Fisher and L. I. Glazman, Transport in a onedimensional Luttinger liquid, Mesoscopic Electron Transport, edited by L. L. Sohn, L. P. Kouwenhoven, and G. Schön (Springer, Dordrecht, 1997), pp. 331-373.

[98] R. Daviet and N. Dupuis, Mott-Glass Phase of a OneDimensional Quantum Fluid with Long-Range Interactions, Phys. Rev. Lett. 125, 235301 (2020). 